\title{
QUANTUM COBORDISMS AND FORMAL GROUP LAWS
}

\author{
TOM COATES AND ALEXANDER GIVENTAL
}

To I. M. Gelfand, who teaches us the unity of mathematics

We would like to congratulate Israel Moiseevich Gelfand on the occasion of his 90th birthday and to thank the organizers P. Etingof, V. Retakh and I. Singer for the opportunity to take part in the celebration. The present paper is closely based on the lecture given by the second author at The Unity of Mathematics Symposium and is based on our joint work in progress on Gromov-Witten invariants with values in complex cobordisms. We will mostly consider here only the simplest example, elucidating one of the key aspects of the theory. We refer the reader to [9] for a more comprehensive survey of the subject and to [5] for all further details. Consider $\overline{\mathcal{M}}_{0, n}, n \geq 3$, the Deligne-Mumford compactification of the moduli space of configurations of $n$ distinct ordered points on the Riemann sphere $\mathbf{C} P^{1}$. Obviously, $\overline{\mathcal{M}}_{0,3}=\mathrm{pt}$, $\overline{\mathcal{M}}_{0,4}=\mathbf{C} P^{1}$, while $\overline{\mathcal{M}}_{0,5}$ is known to be isomorphic to $\mathbf{C} P^{2}$ blown up at 4 points. In general, $\overline{\mathcal{M}}_{0, n}$ is a compact complex manifold of dimension $n-3$, and it makes sense to ask what is the complex cobordism class of this manifold. The Thom ring of complex cobordisms, after tensoring with $\mathbf{Q}$, is known to be isomorphic to $U^{*}=\mathbf{Q}\left[\mathbf{C} P^{1}, \mathbf{C} P^{2}, \ldots\right]$, the polynomial algebra with generators $\mathbf{C} P^{k}$ of degree $-2 k$. Thus our question is to express $\overline{\mathcal{M}}_{0, n}$, modulo the relation of complex cobordism, as a polynomial in complex projective spaces.

This problem can be generalized in the following three directions.

Firstly, one can develop intersection theory for complex cobordism classes from the complex cobordism ring $U^{*}\left(\overline{\mathcal{M}}_{0, n}\right)$. Such intersection numbers take values in the coefficient algebra $U^{*}=U^{*}(\mathrm{pt})$ of complex cobordism theory.

Secondly, one can consider the Deligne-Mumford moduli spaces $\overline{\mathcal{M}}_{g, n}$ of stable $n$-pointed genus- $g$ complex curves. They are known to be compact complex orbifolds, and for an orbifold, one can mimic (as

This material is based upon work supported by the National Science Foundation under Grant No. DMS-0306316. 
explained below) cobordism-valued intersection theory using cohomological intersection theory over $\mathbf{Q}$ against a certain characteristic class of the tangent orbibundle.

Thirdly, one can introduce $[12,3]$ more general moduli spaces $\overline{\mathcal{M}}_{g, n}(X, d)$ of degree- $d$ stable maps from $n$-pointed genus- $g$ complex curves to a compact Kähler (or almost-Kähler) target manifold $X$. One defines Gromov-Witten invariants of $X$ using virtual intersection theory in these spaces (see $[2,8,13,15,16]$ ). Furthermore, using virtual tangent bundles of the moduli spaces of stable maps and their characteristic classes, one can extend Gromov-Witten invariants to take values in the cobordism ring $U^{*}$.

The Quantum Hirzebruch-Riemann-Roch Theorem (see [5]) expresses cobordism-valued Gromov-Witten invariants of $X$ in terms of cohomological ones. Cobordism-valued intersection theory in Deligne-Mumford spaces is included as the special case $X=$ pt. In these notes we will mostly be concerned with this special case, and with curves of genus zero, i.e. with cobordism-valued intersection theory in the manifolds $\overline{\mathcal{M}}_{0, n}$. The cobordism classes of $\overline{\mathcal{M}}_{0, n}$ which we seek are then interpreted as the self-intersections of the fundamental classes.

\section{Cohomological intersection theory on $\overline{\mathcal{M}}_{0, n}$.}

Let $L_{i}, i=1, \ldots, n$, denote the line bundle over $\overline{\mathcal{M}}_{0, n}$ formed by the cotangent lines to the complex curves at the marked point with the index $i$. More precisely, consider the forgetting map $\mathrm{ft}_{n+1}: \overline{\mathcal{M}}_{0, n+1} \rightarrow$ $\overline{\mathcal{M}}_{0, n}$ defined by forgetting the last marked point. Let a point $p \in \overline{\mathcal{M}}_{0, n}$ be represented by a stable genus-zero complex curve $\Sigma$ equipped with the marked points $\sigma_{1}, \ldots, \sigma_{n}$. Then the fiber $\mathrm{ft}_{n+1}^{-1}(p)$ can be canonically identified with $\Sigma$. In particular, the map $\mathrm{ft}_{n+1}$ has $n$ canonical sections $\sigma_{i}$ defined by the marked points, and the diagram formed by the forgetting map and the sections can be considered as the universal family of stable $n$-pointed curves of genus zero. The $L_{i}$ 's are defined as the conormal bundles to the sections $\sigma_{i}: \overline{\mathcal{M}}_{0, n} \rightarrow \overline{\mathcal{M}}_{0, n+1}$ and are often called universal cotangent lines at the marked points.

Put $\psi_{i}=c_{1}\left(L_{i}\right)$ and define the correlator $\left\langle\psi_{1}^{k_{1}}, \ldots, \psi_{n}^{k_{n}}\right\rangle_{0, n}$ to be the intersection index $\int{\overline{\mathcal{M}_{0, n}}}_{1} \psi_{1}^{k_{1}} \ldots \psi_{n}^{k_{n}}$. These correlators are not too hard to compute (see $[17,11]$ ). Moreover, it turns out that intersection theory in the spaces $\overline{\mathcal{M}}_{0, n}$ is governed by the "universal monotone function". Namely, one introduces the genus-0 potential

$$
\mathcal{F}_{0}\left(t_{0}, t_{1}, t_{2}, \ldots\right):=\sum_{n \geq 3} \sum_{k_{1}, \ldots, k_{n} \geq 0}\left\langle\psi_{1}^{k_{1}}, \ldots, \psi_{n}^{k_{n}}\right\rangle_{0, n} \frac{t_{k_{1}} \ldots t_{k_{n}}}{n !} .
$$

It is a formal function of $t_{0}, t_{1}, t_{2}, \ldots$ whose Taylor coefficients are the 
correlators. Then

$$
\mathcal{F}_{0}=\text { critical value of }\left\{\frac{1}{2} \int_{0}^{\tau} Q^{2}(x) d x\right\},
$$

where

$$
Q(x):=-x+t_{0}+t_{1} x+\ldots+t_{n} \frac{x^{n}}{n !}+\ldots
$$

More precisely, the "monotone function" of the variable $\tau$ depending on the parameters $t_{0}, t_{1}, \ldots$ has the critical point $\tau=\tau\left(t_{0}, t_{1}, \ldots\right)$. It can be computed as a formal function of $t_{i}$ 's from the relation $Q(\tau)=0$ which has the form of the "universal fixed point equation":

$$
\tau=t_{0}+t_{1} \tau+\ldots+t_{n} \frac{\tau^{n}}{n !}+\ldots
$$

Then termwise integration in (1) yields the critical value as a formal function of $t_{i}$ 's which is claimed to coincide with $\mathcal{F}_{0}$.

The formula (1) describing $\mathcal{F}_{0}$ can be easily derived by the method of characteristics applied to the following PDE (called the string equation):

$$
\partial_{0} \mathcal{F}_{0}-t_{1} \partial_{t_{0}} \mathcal{F}_{0}-t_{2} \partial_{t_{1}} \mathcal{F}_{0}-\ldots=t_{0}^{2} / 2
$$

The initial condition $\mathcal{F}_{0}=0$ at $t_{0}=0$ holds for dimensional reasons: $\operatorname{dim}_{\mathbf{R}} \overline{\mathcal{M}}_{0, n}<2 n=\operatorname{deg}\left(\psi_{1} \ldots \psi_{n}\right)$. The string equation itself expresses the well-known fact that for $i=1, \ldots, n$, the class $\psi_{i}$ on $\overline{\mathcal{M}}_{0, n+1}$ does not coincide with the pull-back $\mathrm{ft}_{n+1}^{*}\left(\psi_{i}\right)$ of its counterpart from $\overline{\mathcal{M}}_{0, n}$ but differs from it by the class of the divisor $\sigma_{i}\left(\overline{\mathcal{M}}_{0, n}\right)$.

The family of "monotone functions" in (1) can alternatively be viewed as a family of quadratic forms in $Q$ depending on one parameter $\tau$. This leads to the following description of $\mathcal{F}_{0}$ in terms of linear symplectic geometry.

Let $\mathcal{H}$ denote the space of Laurent series $\mathbf{Q}\left(\left(z^{-1}\right)\right)$ in one indeterminate $z^{-1}$. Given two such Laurent series $f, g \in \mathcal{H}$, we put

$$
\Omega(f, g):=\frac{1}{2 \pi i} \oint f(-z) g(z) d z=-\Omega(g, f) .
$$

This pairing is a symplectic form on $\mathcal{H}$, and

$$
f=\ldots-p_{2} z^{-3}+p_{1} z^{-2}-p_{0} z^{-1}+q_{0} z^{0}+q_{1} z^{2}+q_{2} z^{2}+\ldots
$$

is a Darboux coordinate system.

The subspaces $\mathcal{H}_{+}:=\mathrm{Q}[z]$ and $\mathcal{H}_{-}:=z^{-1} \mathbf{Q}\left[\left[z^{-1}\right]\right]$ form a Lagrangian polarization of $(\mathcal{H}, \Omega)$ and identify the symplectic space with $T^{*} \mathcal{H}_{+}$. Next, we consider $\mathcal{F}_{0}$ as a formal function on $\mathcal{H}_{+}$near the shifted origin $-z$ by putting:

$$
q_{0}+q_{1} z+\ldots+q_{n} z^{n}+\ldots=-z+t_{0}+t_{1} z+\ldots+t_{n} z^{n}+\ldots
$$


This convention - called the dilaton shift - makes many ingredients of the theory homogeneous, as is illustrated by the following examples: the vector field on the L.H.S. of the string equation is $-\sum q_{k+1} \partial_{q_{k}}$; $Q(x)$ in (1) becomes $\sum q_{k} x^{k} / k$ !; $\mathcal{F}_{0}$ becomes homogeneous of degree 2 , i.e. $\sum q_{k} \partial_{q_{k}} \mathcal{F}_{0}=2 \mathcal{F}_{0}$. Furthermore, we define a (formal germ of a) Lagrangian submanifold $\mathcal{L} \subset \mathcal{H}$ as the graph of the differential of $\mathcal{F}_{0}$ :

$$
\mathcal{L}=\left\{(\mathbf{p}, \mathbf{q}) \mid \mathbf{p}=d_{z+\mathbf{q}} \mathcal{F}_{0}\right\} \subset \mathcal{H} \cong T^{*} \mathcal{H}_{+} .
$$

Then $\mathcal{L} \subset \mathcal{H}$ is the Lagrangian cone

$$
\mathcal{L}=\cup_{\tau} e^{\tau / z} z \mathcal{H}_{+}=\left\{z e^{\tau / z} \mathbf{q}(z) \mid \mathbf{q} \in \mathbf{Q}[z], \tau \in \mathbf{Q}\right\} .
$$

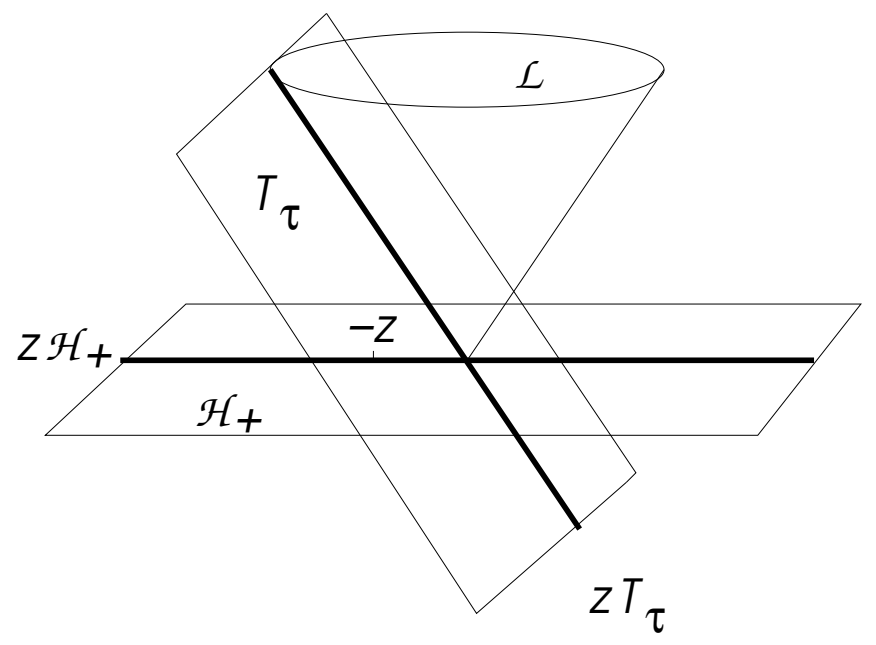

Figure 1. The Lagrangian cone $\mathcal{L}$.

We make a few remarks about this formula. The tangent spaces to the cone $\mathcal{L}$ form a 1 -parametric family $T_{\tau}=e^{\tau / z} \mathcal{H}_{+}$of semi-infinite Lagrangian subspaces. These are graphs of the differentials of the quadratic forms from (1). The subspaces $T_{\tau}$ are invariant under multiplication by $z$ and form a variation of semi-infinite Hodge structures in the sense of S. Barannikov [1]. The graph $\mathcal{L}$ of $d \mathcal{F}_{0}$ is therefore the envelope to such a variation. Moreover, the tangent spaces $T_{\tau}$ of $\mathcal{L}$ are tangent to $\mathcal{L}$ exactly along $z T_{\tau}$. All these facts, properly generalized, remain true in genus-zero Gromov-Witten theory with a non-trivial target space $X$ (see [9]).

\section{Complex cobordism theory.}

Complex cobordism is an extraordinary cohomology theory $U^{*}(\cdot)$ defined in terms of homotopy classes $\Pi$ of maps to the spectrum $M U(k)$ 
of the Thom spaces of universal $U_{k / 2}$-bundles:

$$
U^{n}(B)=\lim _{k \rightarrow \infty} \Pi\left(\Sigma^{k} B, M U(n+k)\right) .
$$

The dual homology theory, called bordism, can be described geometrically as

$$
U_{n}(B):=\left\{\operatorname{maps} Z^{n} \rightarrow B\right\} / \text { bordism },
$$

where $Z^{n}$ is a compact stably almost complex manifold of real dimension $n$, and the bordism manifold should carry a stably almost complex structure compatible in the obvious sense with that of the boundary.

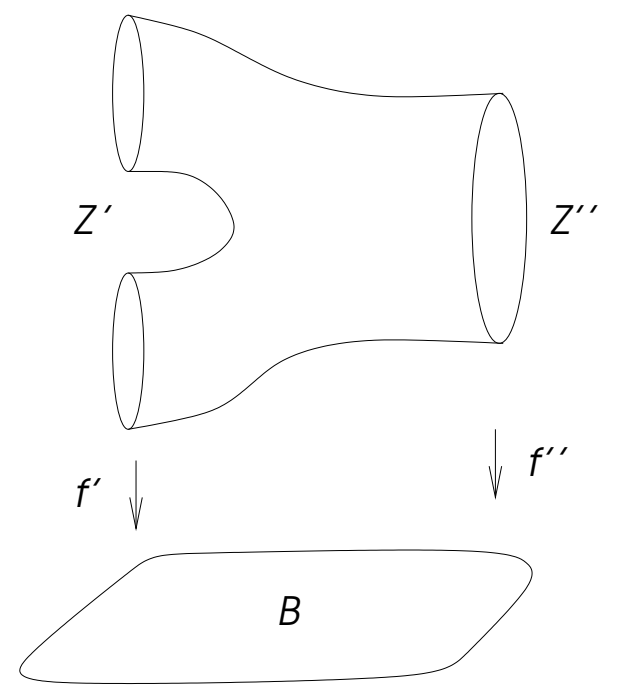

Figure 2. A bordism between $\left(Z^{\prime}, f^{\prime}\right)$ and $\left(Z^{\prime \prime}, f^{\prime \prime}\right)$.

When $B$ itself is a compact stably almost complex manifold of real dimension $m$, the celebrated Pontryagin-Thom construction identifies $U^{n}(B)$ with $U_{m-n}(B)$ and plays the role of the Poincaré isomorphism.

One can define characteristic classes of complex vector bundles which take values in complex cobordism. The splitting principle in the theory of vector bundles identifies $U^{*}\left(B U_{n}\right)$ with the symmetric part of $U^{*}\left(\left(B U_{1}\right)^{n}\right)$. Thus to define cobordism-valued Chern classes it suffices to describe the first Chern class $u \in U^{2}\left(\mathbf{C} P^{\infty}\right)$ of the universal complex line bundle. By definition, the first Chern class of $\mathcal{O}(1)$ over $\mathbf{C} P^{N}$ is Poincaré-dual to the embedding $\mathbf{C} P^{N-1} \rightarrow \mathbf{C} P^{N}$ of a hyperplane section.

The operation of tensor product of line bundles defines a formal commutative group law on the line with co-ordinate $u$. Namely, the tensor product of the line bundles with first Chern classes $v$ and $w$ has the 
first Chern class $u=F(v, w)=v+w+\ldots$. The group properties follow from associativity of tensor product and invertibility of line bundles.

Henceforth we use the notation $U^{*}(\cdot)$ for the cobordism theory tensored with $\mathbf{Q}$.

Much as in complex K-theory, there is the Chern-Dold character which provides natural multiplicative isomorphisms

$$
\mathrm{Ch}: U^{*}(B) \rightarrow H^{*}\left(B, U^{*}\right) .
$$

Here $U^{*}=U^{*}(p t)$ is the coefficient ring of the theory and is isomorphic to the polynomial algebra on the generators of degrees $-2 k$ Poincarédual to the bordism classes $\left[\mathbf{C} P^{k}\right]$. We refer to, say, [4] for the construction. The Chern-Dold character applies, in particular, to the universal cobordism-valued first Chern class of complex line bundles:

$$
\operatorname{Ch}(u)=u(z)=z+a_{1} z^{2}+a_{2} z^{3}+\ldots,
$$

where $z$ is the cohomological first Chern of the universal line bundle $\mathcal{O}(1)$ over $\mathbf{C} P^{\infty}$, and $\left\{a_{k}\right\}$ is another set of generators in $U^{*}$. The series $u(z)$ can be interpreted as an isomorphism between the formal group corresponding to complex cobordism and the additive group $(x, y) \mapsto$ $x+y$ :

$$
F(v, w)=u(z(v)+z(w))
$$

where $z(\cdot)$ is the series inverse to $u(z)$. This is known as the logarithm of the formal group law and takes the form

$$
z=u+\left[\mathbf{C} P^{1}\right] \frac{u^{2}}{2}+\left[\mathbf{C} P^{2}\right] \frac{u^{3}}{3}+\left[\mathbf{C} P^{3}\right] \frac{u^{4}}{4}+\ldots
$$

Much as in K-theory, one can compute push-forwards in complex cobordism in terms of cohomology theory. In particular, for a map $\pi: B \rightarrow$ pt from a stably almost complex manifold $B$ to a point, we have the Hirzebruch - Riemann - Roch formula

$$
\pi_{*}^{U}(c)=\int_{B} \operatorname{Ch}(c) \operatorname{Td}\left(T_{B}\right) \in U^{*} \quad \forall c \in U^{*}(B)
$$

where $\operatorname{Td}\left(T_{B}\right)$ is the Todd genus of the tangent bundle. By definition, the push-forward $\pi_{*}^{U}$ of the cobordism class $c$ represented by the Poincaré-dual bordism class represented by $Z \rightarrow B$ is the class of the manifold $Z$ in $U^{*}$. In cobordism theory, the Todd genus is the universal cohomology-valued stable multiplicative characteristic class of complex vector bundles:

$$
\operatorname{Td}(\cdot)=\exp \sum_{m=1}^{\infty} s_{m} \operatorname{ch}_{m}(\cdot)
$$


where $\mathbf{s}=\left(s_{1}, s_{2}, s_{3}, \ldots\right)$ is yet another set of generators of the coefficient algebra $U^{*}$. To find out which one, use the fact that on the universal line bundle $\operatorname{Td}\left(\mathcal{O}(1)=\frac{z}{u(z)}\right.$ and so

$$
\exp \sum_{m=1}^{\infty} s_{m} \frac{z^{m}}{m !}=\sum_{k \geq 0}\left[\mathbf{C} P^{k}\right] \frac{u^{k}(z)}{k+1}
$$

\section{Cobordism-valued intersection theory of $\overline{\mathcal{M}}_{0, n}$.}

Let $\Psi_{i} \in U^{*}\left(\overline{\mathcal{M}}_{0, n}\right), i=1, \ldots, n$, denote the cobordism-valued first Chern classes of the universal cotangent lines $L_{i}$ over $\overline{\mathcal{M}}_{0, n}$. We introduce the correlators

$$
\left\langle\Psi_{1}^{k_{1}}, \ldots, \Psi_{n}^{k_{n}}\right\rangle_{0, n}^{U}:=\pi_{*}^{U}\left(\Psi_{1}^{k_{1}} \ldots \Psi_{n}^{k_{n}}\right)
$$

and the genus- 0 potential

$$
\mathcal{F}_{0}^{U}\left(t_{0}, t_{1}, \ldots\right):=\sum_{n \geq 3} \sum_{k_{1}, \ldots, k_{n} \geq 0}\left\langle\Psi_{1}^{k_{1}}, \ldots, \Psi_{n}^{k_{n}}\right\rangle_{0, n} \frac{t_{k_{1}} \ldots t_{k_{n}}}{n !}
$$

which take values in the coefficient ring $U^{*}$ of complex cobordism theory. In particular, the generating function $F_{0}^{U}:=\sum_{n \geq 3}\left[\overline{\mathcal{M}}_{0, n}\right] t_{0}^{n} / n$ ! for the bordism classes of $\overline{\mathcal{M}}_{0, n}$ is obtained from $\mathcal{F}_{0}^{U}$ by putting $t_{1}=t_{2}=$ $\ldots=0$. Our present goal is to compute $\mathcal{F}_{0}^{U}$.

According to the Hirzebruch-Riemann-Roch formula (4), the computation can be reduced to cohomological intersection theory in $\overline{\mathcal{M}}_{0, n}$ between $\psi$-classes and characteristic classes of the tangent bundle. In the more general context of (higher genus) Gromov-Witten theory, one can consider intersection numbers involving characteristic classes of the virtual tangent bundles of moduli spaces of stable maps as a natural generalization of "usual" Gromov-Witten invariants. It turns out that it is possible to express these generalized Gromov-Witten invariants in terms of the usual ones, but the explicit formulas seem unmanageable unless one interprets the generalized invariants as the R.H.S. of the Hirzebruch-Riemann-Roch formula in complex cobordism theory. This interpretation of the (cohomological) intersection theory problem in terms of cobordism theory dictates a change of the symplectic formalism described in Section 1, and this change alone miraculously provides a radical simplification of the otherwise unmanageable formulas. In this section, we describe how this happens in the example of the spaces $\overline{\mathcal{M}}_{0, n}$.

Let $U$ denote the formal series completion of the algebra $U^{*}$ in the topology defined by the grading. Introduce the symplectic space ${ }^{1}$

\footnotetext{
${ }^{1}$ We continue to call it "space" although it is a $U$-module.
} 
$\left(\mathcal{U}, \Omega^{U}\right)$ defined over $U$. Let $\mathcal{U}$ denote the space of Laurent series $\sum_{k \in \mathbf{Z}} f_{k} u^{k}$ with coefficients $f_{k} \in U$ which are possibly infinite in both directions but satisfy the condition $\lim _{k \rightarrow+\infty} f_{k}=0$ in the topology of $U$. We will call such series convergent and write $\mathcal{U}=U\left\{\left\{u^{-1}\right\}\right\}$. For $f, g \in \mathcal{U}$ we define $\Omega^{U}(f, g) \in U$ by

$$
\Omega^{U}(f, g):=\frac{1}{2 \pi i} \sum_{n=0}^{\infty}\left[\mathbf{C} P^{n}\right] \oint f\left(u^{*}\right) g(u) u^{n} d u
$$

where $u^{*}$ is the inverse to $u$ in the formal group law described in Section 2. Using the formal group isomorphism $u=u(z)$ we find $u^{*}(z)=$ $u(-z(u))$. The formula (3) for the logarithm $z=z(u)$ shows that our integration measure $\sum\left[\mathbf{C} P^{n}\right] u^{n} d u$ coincides with $d z$. Thus the following quantum Chern character $\mathrm{qCh}$ is a symplectic isomorphism, $\mathrm{qCh}^{*} \Omega=\Omega^{U}$ :

$$
\mathrm{qCh}: \mathcal{U} \mapsto \mathcal{H} \hat{\otimes} U, \sum_{k \in \mathbf{Z}} f_{k} u^{k} \mapsto \sum_{k \in \mathbf{Z}} f_{k} u^{k}(z)
$$

The "hat" over the tensor product sign indicates the necessary completion by convergent Laurent series.

The next step is to define a Lagrangian submanifold $\mathcal{L}^{U} \subset \mathcal{U}$ as the graph of the differential of the generating function $\mathcal{F}_{0}^{U}$. This requires a Lagrangian polarization $\mathcal{U}=\mathcal{U}_{+} \oplus \mathcal{U}_{-}$. We define $\mathcal{U}_{+}=U\{u\}$ to be the space of convergent power series. However there is no reason for the opposite subspace $u^{-1} U\left\{\left\{u^{-1}\right\}\right\}$ to be Lagrangian relative to $\Omega^{U}$. We need a more conceptual construction of the polarization. This is provided by the following residue formula:

$$
\frac{1}{2 \pi i} \oint \frac{d z}{u(z-x) u(-z-y)}=\left\{\begin{array}{ccc}
+\frac{1}{u(-x-y)} & \text { if } & |x|<|z|<|y| \\
-\frac{1}{u(-x-y)} & \text { if } & |y|<|z|<|x| \\
0 & \text { otherwise }
\end{array}\right.
$$

The integrand here has the first order poles at $z=x$ and $z=-y$, and the integral depends on the property of the contour to enclose both, neither or one of them.

One can pick a topological basis $\left\{u_{k}(z) \mid k=0,1,2, \ldots\right\}$ in the $U$ module $U\{u\}={ }_{\mathrm{qCh}} U\{z\}\left(\right.$ e.g. $1, u(z), u(z)^{2}, \ldots$ or $\left.1, z, z^{2}, \ldots\right)$ and expand $u(x+y)$ in the region $|x|<|y|$ as

$$
\frac{1}{u(-x-y)}=\sum_{k=0}^{\infty} u_{k}(x) v_{k}(y)
$$


where the $v_{k}$ are convergent Laurent series in $y^{-1}$ (or equivalently in $u^{-1}$ ). The residue formulas then show that

$$
\begin{aligned}
\sum_{l, m \geq 0} \Omega^{U}\left(v_{l}, u_{m}\right) u_{l}(x) v_{m}(y) & =\sum_{k \geq 0} u_{k}(x) v_{k}(y) \\
\sum_{l, m \geq 0} \Omega^{U}\left(u_{l}, u_{m}\right) v_{l}(x) v_{m}(y) & =0 \\
\sum_{l, m \geq 0} \Omega^{U}\left(v_{l}, v_{m}\right) u_{l}(x) u_{m}(y) & =0
\end{aligned}
$$

or in other words, $\left\{v_{0}, u_{0} ; v_{1}, u_{1} ; \ldots\right\}$ is a Darboux basis in $\left(\mathcal{U}, \Omega^{U}\right)$. For example, in cohomology theory (which we recover by setting $a_{1}=a_{2}=$ $\ldots=0)$, the Darboux basis $\left\{-z^{-1}, 1 ; z^{-2}, z ;-z^{-3}, z^{2} ; \ldots\right\}$ is obtained from the expansion

$$
\frac{1}{-x-y}=\sum_{k \geq 0} x^{k}(-y)^{-1-k} .
$$

We define $\mathcal{U}_{-}$to be the Lagrangian subspace spanned by $\left\{v_{k}\right\}$ :

$$
\mathcal{U}_{-}:=\left\{\sum_{k \geq 0} f_{-k} v_{k}, \quad f_{-k} \in U\right\} .
$$

A different choice $u_{k}^{\prime}=\sum c_{k l} u_{l}$ of basis in $\mathcal{U}_{+}$yields

$$
\sum_{l} u_{l}(x) v_{l}(y)=\sum_{k} u_{k}^{\prime}(x) v_{k}^{\prime}(y)=\sum_{k l} u_{l}(x) c_{k l} v_{k}^{\prime}(y),
$$

so that $v_{l}=\sum_{l} c_{k l} v_{k}^{\prime}$. Thus the subspace $\mathcal{U}_{-}$spanned by $v_{k}^{\prime}$ remains the same.

As before, the Lagrangian polarization $\mathcal{U}=\mathcal{U}_{+} \oplus \mathcal{U}_{-}$identifies $\left(\mathcal{U}, \Omega^{U}\right)$ with $T^{*} \mathcal{U}_{+}$. We define a (formal germ of a) Lagrangian section

$$
\mathcal{L}^{U}:=\left\{(\mathbf{p}, \mathbf{q}) \in T^{*} \mathcal{U}_{+} \mid \mathbf{p}=d_{-u^{*}+\mathbf{q}} \mathcal{F}^{U}\right\} \subset \mathcal{U} .
$$

Note that the dilaton shift is defined by the formula

$$
q_{0}+q_{1} u+q_{2} u^{2}+\ldots=u^{*}(u)+t_{0}+t_{1} u+t_{2} u^{2}+\ldots
$$

involving the inversion $u^{*}(u)$ in the formal group. This reduces to the dilaton shift $-z$ in the cohomological theory when we set $a_{1}=a_{2}=$ $\ldots=0$.

Our goal is to express the image $\mathrm{qCh}\left(\mathcal{L}^{U}\right)$ in terms of the Lagrangian cone

$$
\mathcal{L}=\cup_{\tau \in U} z e^{\tau / z}\left(\mathcal{H}_{+} \hat{\otimes} U\right) \subset \mathcal{H} \hat{\otimes} U
$$

defined by cohomological intersection theory in $\overline{\mathcal{M}}_{0, n}$. The answer is given by the following theorem (see [5]). 
Theorem. $\mathrm{qCh}\left(\mathcal{L}^{U}\right)=\mathcal{L}$.

Corollary. $\mathcal{L}^{U}$ is a Lagrangian cone with the property that its tangent spaces $T$ are tangent to $\mathcal{L}^{U}$ exactly along $u T$.

\section{Extracting intersection indices.}

In this section, we unpack the information hidden in the abstract formulation of Theorem to compute the fundamental classes $\left[\overline{\mathcal{M}}_{0, n}\right]$ in $U^{*}$. We succeed for small values of $n$, but things become messy soon. A lesson to learn is that there is no conceptual advantage in doing this, and that Theorem as stated provides a better way of representing the answers.

The Theorem together with our conventions $(6),(7)$ on the polarization and dilaton shift encode cobordism-valued intersection indices in $\overline{\mathcal{M}}_{0, n}$. We may view $\mathrm{qCh}\left(\mathcal{U}_{-}\right)$as a family of Lagrangian subspaces depending on the parameters $\left(a_{1}, a_{2}, \ldots\right)$. The dilaton shift $u(-z)$ can be interpreted in the a similar parametric sense. First, the value of $\mathcal{F}_{0}^{U}$ considered as a function on the conical graph $\mathcal{L}^{U}$ of $d \mathcal{F}_{0}^{U}$ is equal at a point $f=\sum\left(p_{k} v_{k}+q_{k} u_{k}\right)$ to the value of the quadratic form

$$
\frac{1}{2} \sum_{k \geq 0} p_{k} q_{k}=\frac{1}{2} \sum_{k \geq 0} q_{k} \Omega^{U}\left(f, u_{k}\right) .
$$

The projection $\sum q_{k} u_{k}$ of $f$ to $\mathcal{U}_{+}$along $\mathcal{U}_{-}$is

$$
\sum_{k \geq 0} q_{k} u_{k}(x)=\sum_{k \geq 0} u_{k}(x) \Omega^{U}\left(\sum v_{k}, f\right)=\frac{1}{2 \pi i} \oint_{|x|<|z|} \frac{f(z) d z}{u(z-x)} .
$$

Combining, we find that the value of $\mathcal{F}_{0}^{U}$ at the point $f=-z e^{\tau / z} \mathbf{q}(z) \in$ $\mathcal{L}$ is given by the double residue

$$
\frac{1}{8 \pi^{2}} \oint_{|x|<|z|} \frac{d z d x}{u(z+x)} x z \mathbf{q}(x) \mathbf{q}(z) e^{\left[\tau\left(\frac{1}{x}+\frac{1}{z}\right)\right]} .
$$

The integral vanishes at $\tau=0$. Differentiating in $\tau$ brings down the factor $(z+x) / z x$. We expand $(z+x) / u(z+x)=1+\sum_{k>0} b_{k}(z+x)^{k}$, where $\left(b_{1}, b_{2}, \ldots\right)$ is one more set of generators in $U^{*}$ — "the Bernoulli polynomials". Each factor $(z+x)$ can be replaced by $z x$ and differentiation in $\tau$. Using this we express the double integrals via the product of single integrals. After some elementary calculations we obtain the result in the form

$$
-\frac{1}{2} \int_{0}^{\tau}\left[Q^{(-1)}(y)\right]^{2} d y-\frac{1}{2} \sum_{k>0} b_{k} \frac{d^{k-1}}{d \tau^{k-1}}\left[Q^{(-1-k)}(\tau)\right]^{2},
$$




$$
\text { where } Q^{(-l)}(\tau)=\left(\frac{d}{d \tau}\right)^{-l} Q(\tau):=\sum_{m \geq 0} q_{m} \frac{\tau^{m+l}}{(m+l) !} .
$$

Thus (8) gives the value of the potential $\mathcal{F}_{0}^{U}$ at the point $\left(t_{0}, t_{1}, \ldots\right)$ which is computed as

$$
t_{0}+t_{1} x+t_{2} x^{2}+\ldots=-u(-x)-\frac{1}{2 \pi i} \oint_{|x|<|z|} \frac{z e^{\tau / z} \mathbf{q}(z) d z}{u(z-x)} .
$$

To evaluate the latter expression we write $1 /(z-x)=\sum_{r>0} x^{s-1} z^{s}$, expand $(z-u) / u(z-u)$ as before and use the binomial formula for $(z-x)^{r}$. The resulting expression is

$$
-u(-x)-\sum_{k \geq 0} x^{k} Q^{(k-1)}(\tau)-\sum_{k \geq 0}(-x)^{k} \sum_{l \geq 0} Q^{(-2-l)}(\tau)\left(\begin{array}{c}
k+l \\
k
\end{array}\right) b_{k+l+1} .
$$

It leads to the sequence of equations (we put $a_{-1}=0, a_{0}=b_{0}=1$ ):

$$
t_{k}+(-1)^{k} a_{k-1}+\sum_{l \geq 0} b_{l} Q^{(k-1-l)}(\tau) \frac{(1-l)(2-l) \ldots(k-l)}{k !}=0 .
$$

These equations are homogeneous relative to the grading

$$
\operatorname{deg} \tau=1, \operatorname{deg} t_{k}=1-k, \operatorname{deg} a_{k}=\operatorname{deg} b_{k}=\operatorname{deg} q_{k}=-k
$$

and need to be solved for $q_{0}, q_{1}, \ldots \in U^{*}$ and $\tau \in U^{*}\left[\left[t_{0}, t_{1}, \ldots\right]\right]$.

With the aim of computing $F_{0}^{U}(t):=\left.\mathcal{F}_{0}^{U}\right|_{t_{0}=t, t_{1}=t_{2}=\ldots=0}$ modulo $\left(t^{7}\right)$ we work modulo elements in $U^{*}$ of degree $<-3$ and set up the equations (9) with $k=0,1,2,3,4$ :

$$
\begin{aligned}
& t+q_{0} \tau \quad+q_{1} \tau^{2} / 2 \quad+q_{2} \tau^{3} / 6 \quad+q_{3} \tau^{4} / 24 \\
& +b_{1}\left(q_{0} \tau^{2} / 2+q_{1} \tau^{3} / 6 \quad+q_{2} \tau^{4} / 24\right) \\
& +b_{2}\left(q_{0} \tau^{3} / 6+q_{1} \tau^{4} / 24\right) \\
& +b_{3} q_{0} \tau^{4} / 24=0 \\
& q_{0}-1 \quad+q_{1} \tau \quad+q_{2} \tau^{2} / 2 \quad+q_{3} \tau^{3} / 6 \\
& -b_{2}\left(q_{0} \tau^{2} / 2+q_{1} \tau^{3} / 6\right) \\
& -2 b_{3} q_{0} \tau^{3} / 6=0 \\
& a_{1}+q_{1} \quad+q_{2} \tau \quad+q_{3} \tau^{2} / 2 \\
& +b_{3} q_{0} \tau^{2} / 2=0 \\
& -a_{2}+q_{2} \quad+q_{3} \tau=0 \\
& a_{3}+q_{3} \quad=0
\end{aligned}
$$

We solve this system consecutively modulo elements in $U^{*}$ of degree $n<0,-1,-2,-3$. The relation $\sum b_{k} x^{k}=\left(\sum a_{k} x^{k}\right)^{-1}$ implies

$$
b_{1}=-a_{1}, b_{2}=a_{1}^{2}-a_{2}, b_{3}=2 a_{1} a_{2}-a_{3}-a_{1}^{3} .
$$


Taking this into account we find (using MAPLE):

$$
\begin{array}{llll}
\tau=-t & & +\left(a_{1}^{2} / 3-a_{2} / 2\right) t^{3} & +\left(7 a_{3}-11 a_{1} a_{2}+5 a_{1}^{3}\right) t^{4} / 12 \\
q_{0}=1 & -a_{1} t & +a_{1}^{2} t^{2} / 2 & +\left(a_{1}^{3}-a_{3}-2 a_{1} a_{2} / 3\right) t^{3} \\
q_{1}= & -a_{1} & +a_{2} t & +\left(a_{3}-a_{1} a_{2}+a_{1}^{3} / 2\right) t^{2} \\
q_{2}= & & a_{2} & -a_{3} t \\
q_{3}= & & & -a_{3}
\end{array}
$$

From (8), we find $F_{0}^{U}(t)$ modulo $\left(t^{7}\right)$ :

$$
\begin{gathered}
F_{0}^{U}=\bmod \left(t^{7}\right)-\frac{q_{0}^{2} \tau^{3}}{6}-\frac{q_{0} q_{1} \tau^{4}}{8}-\frac{q_{1}^{2} \tau^{5}}{40}-\frac{q_{0} q_{2} \tau^{5}}{30}-\frac{q_{1} q_{2} \tau^{6}}{72}-\frac{q_{0} q_{3} \tau^{6}}{144} \\
-\frac{b_{1}}{2}\left(\frac{q_{0} \tau^{2}}{2}+\frac{q_{1} \tau^{3}}{6}+\frac{q_{2} \tau^{4}}{24}\right)^{2}-b_{2}\left(\frac{q_{0} \tau^{2}}{2}+\frac{q_{1} \tau^{3}}{6}\right)\left(\frac{q_{0} \tau^{3}}{6}+\frac{q_{1} \tau^{4}}{24}\right)-\frac{7 b_{3} q_{0}^{2} \tau^{6}}{144} .
\end{gathered}
$$

Substituting the previous formulas into this mess we compute

$$
F_{0}^{U}=\frac{t^{3}}{6}-a_{1} \frac{t^{4}}{12}+\left(6 a_{2}-2 a_{1}^{2}\right) \frac{t^{5}}{120}+\left(10 a_{1}^{3}-10 a_{1} a_{2}-34 a_{3}\right) \frac{t^{6}}{720}+O\left(t^{7}\right) .
$$

Expressing $u(x)=x+a_{1} x^{2}+a_{2} x^{3}+a_{3} x^{4}+\ldots$ as the inverse function to $x(u)=u+p_{1} u^{2} / 2+p_{2} u^{3} / 3+p_{3} u^{4} / 4+\ldots$, we find

$$
a_{1}=-p_{1} / 2, a_{2}=p_{1}^{2} / 2-p_{2} / 3, a_{3}=-5 p_{1}^{3} / 8+5 p_{1} p_{2} / 6-p_{3} / 4
$$

and finally arrive at

$$
F_{0}^{U}=\frac{t^{3}}{6}+p_{1} \frac{t^{4}}{24}+\left(4 p_{1}^{2}-3 p_{2}\right) \frac{t^{5}}{120}+\left(\frac{45}{2} p_{1}^{3}-30 p_{1} p_{2}+\frac{17}{2} p_{3}\right) \frac{t^{6}}{720}+O\left(t^{7}\right) .
$$

The coefficients in this series mean that $\left[\overline{\mathcal{M}}_{0,3}\right]=[\mathrm{pt}],\left[\overline{\mathcal{M}}_{0,4}\right]=\left[\mathbf{C} P^{1}\right]$, that each blow-up of a complex surface $M\left(=\mathbf{C} P^{2}\right.$ in our case $)$ adds $\left[\mathbf{C} P^{1} \times \mathbf{C} P^{1}\right]-\left[\mathbf{C} P^{2}\right]$ to its cobordism class, ${ }^{2}$ and that

$$
\left[\overline{\mathcal{M}}_{0,6}\right]=\frac{45}{2}\left[\mathbf{C} P^{1} \times \mathbf{C} P^{1} \times \mathbf{C} P^{1}\right]-30\left[\mathbf{C} P^{1} \times \mathbf{C} P^{2}\right]+\frac{17}{2}\left[\mathbf{C} P^{3}\right] .
$$

The coefficients' sum $(45 / 2-30+17 / 2)$ yields the arithmetical genus of $\left[\overline{\mathcal{M}}_{0,6}\right]$ (which is equal to 1 for all $\overline{\mathcal{M}}_{0, n}$ since they are rational manifolds).

\section{Quantum Hirzebruch-Riemann-Roch Theorem.}

Assuming that the reader is familiar with generalities of GromovWitten theory we briefly explain below how the Theorem of Section 3 generalizes to higher genera and arbitrary target spaces.

\footnotetext{
${ }^{2}$ This is not hard to verify by studying the behavior under blow-ups of the Chern characteristic numbers $\int_{M} c_{1}\left(T_{M}\right)^{2}$ and $\left.\int_{M} c_{2}\left(T_{M}\right)\right)$, or simply using the fact that $\mathbf{C} P^{1} \times \mathbf{C} P^{1}$ is obtained from $\mathbf{C} P^{2}$ by two blow-ups and one blow-down.
} 
Let $X$ be a compact almost Kähler manifold. Gromov-Witten invariants of $X$ with values in complex cobordism are defined by

$$
\left\langle\phi_{1} \Psi_{1}^{k_{1}}, \ldots, \phi_{n} \Psi_{n}^{k_{n}}\right\rangle_{g, n}^{X, d}:=\int_{\left[\overline{\mathcal{M}}_{g, n}(X, d)\right]} \operatorname{Td}\left(T_{g, n}^{X, d}\right) \prod_{i=1}^{n} \operatorname{ev}_{i}^{*}\left(\operatorname{Ch}\left(\phi_{i}\right)\right) u\left(\psi_{i}\right)^{k_{i}} .
$$

Here $\left[\overline{\mathcal{M}}_{g, n}(X, d)\right]$ is the virtual fundamental class of the moduli space of degree- $d$ stable pseudo-holomorphic maps to $X$ from genus- $g$ curves with $n$ marked points, $\phi_{i} \in U^{*}(X)$ are cobordism classes on $X, \mathrm{ev}_{i}$ : $\overline{\mathcal{M}}_{g, n}(X, d) \rightarrow X$ are evaluation maps at the marked points, $\psi_{i}$ are (cohomological) first Chern classes of the universal cotangent line bundles over $\overline{\mathcal{M}}_{g, n}(X, d)$, and $T_{g, n}^{X, d}$ is the virtual tangent bundle of $\overline{\mathcal{M}}_{g, n}(X, d)$.

The genus- $g$ potential of $X$ is the generating function

$$
\mathcal{F}_{g, X}^{U}:=\sum_{n, d} \frac{Q^{d}}{n !} \sum_{k_{1}, \ldots, k_{n} \geq 0}\left\langle\phi_{\alpha_{1}} \Psi_{1}^{k_{1}}, \ldots, \phi_{\alpha_{n}} \Psi_{n}^{k_{n}}\right\rangle_{g, n}^{X, d} t_{k_{1}}^{\alpha_{1}} \ldots t_{k_{n}}^{\alpha_{n}},
$$

where $\left\{\phi_{\alpha}\right\}$ is a basis of the free $U^{*}(\mathrm{pt})$-module $U^{*}(X), Q^{d}$ is the monomial in the Novikov ring representing the degree $d \in H_{2}(X)$, and $\left\{t_{k}^{\alpha}, k=0,1,2, \ldots\right\}$ are formal variables. The total potential of $X$ is the expression

$$
\mathcal{D}_{X}^{U}:=\exp \left(\sum_{g=0}^{\infty} \hbar^{g-1} \mathcal{F}_{g, X}^{U}\right)
$$

The exponent is a formal function (with values in a coefficient ring that includes $U^{*}(\mathrm{pt})$, the Novikov ring and Laurent series in $\hbar$ ) on the space of polynomials $\mathbf{t}:=t_{0}+t_{1} \Psi+t_{2} \Psi^{2}+\ldots$ with vector coefficients $t_{k}=\sum_{\alpha} t_{k}^{\alpha} \phi_{\alpha}$.

We prefer to consider $\mathcal{D}_{X}^{U}$ as a family of formal expressions depending on the sequence of parameters $\mathbf{s}=\left(s_{1}, s_{2}, \ldots\right)$ - generators of $U^{*}(\mathrm{pt})$ featuring in the definition of the Todd genus $T d(\cdot)=\exp \sum s_{k} \operatorname{ch}_{k}(\cdot)$. The specialization $\mathbf{s}=0$ yields the total potential $\mathcal{D}_{X}$ of cohomological Gromov-Witten theory on $X$. Our goal is to express $\mathcal{D}_{X}^{U}$ in terms of $\mathcal{D}_{X}$.

To formulate our answer we interpret $\mathcal{D}_{X}^{U}$ as an asymptotic element of some Fock space, the quantization of an appropriate symplectic space defined as follows. Let $U$ now denote the super-space $U^{*}(X)$ tensored with $\mathbf{Q}$ and with the Novikov ring and completed, as before, in the formal series topology of the coefficient ring $U^{*}(\mathrm{pt})$. Let $(a, b)^{U}$ denote the cobordism-valued Poincaré pairing:

$$
(a, b)^{U}:=\pi_{*}^{U}(a b) \in U
$$


where $\pi: X \rightarrow$ pt. We put

$$
\mathcal{U}:=U\left\{\left\{u^{-1}\right\}\right\}, \Omega^{U}(f, g)=\frac{1}{2 \pi i} \sum_{n \geq 0}\left[\mathbf{C} P^{n}\right] \oint\left(f\left(u^{*}\right), f(u)\right)^{U} u^{n} d u
$$

and define a Lagrangian polarization of $\left(\mathcal{U}, \Omega^{U}\right)$ using $(6)$ :

$$
\mathcal{U}_{+}=U\{u\}, \quad \mathcal{U}_{-}:=\left\{\sum_{k \geq 0} p_{k} v_{k} \mid p_{k} \in U\right\} .
$$

Using the dilaton shift $\mathbf{q}(u)=1 u^{*}+\mathbf{t}(u)$ (where 1 is the unit element of $U$ ) we identify $\mathcal{D}_{X}$ with an asymptotic function of $\mathbf{q} \in \mathcal{U}_{+}$. By definition, this means that the exponent in $\mathcal{D}_{X}$ is a formal function of $\mathbf{q}$ near the shifted origin. The Heisenberg Lie algebra of $\left(\mathcal{U}, \Omega^{U}\right)$ acts on functions on $\mathcal{U}$ invariant under translations by $\mathcal{U}_{-}$, and this action extends to the (non-linear) space of asymptotic functions.

In the specialization $\mathbf{s}=0$ the above structure degenerates into its cohomological counterpart introduced in [10]: $(\mathcal{H}, \Omega)=T^{*} \mathcal{H}_{+}$ where $\mathcal{H}:=H\left(\left(z^{-1}\right)\right)$ consists of Laurent series in $z^{-1}$ with vector coefficients in the cohomology super-space $H=H^{*}(X, \mathbf{Q})$ tensored with the Novikov ring, $\mathcal{H}_{+}=H[z], \mathcal{H}_{-}=z^{-1} H\left[\left[z^{-1}\right]\right], \Omega(f, g)=$ $(2 \pi i)^{-1} \oint(f(-z), g(z)) d z$, and $(a, b):=\int_{X} a b$ is the cohomological Poincaré pairing. The total potential $\mathcal{D}_{X}$ is identified via the dilaton shift $\mathbf{q}(z)=\mathbf{t}(z)-z$ with an asymptotic element of the corresponding Fock space.

The quantum Chern-Dold character of Section 3 is generalized in the following way

$$
\mathrm{qCh}\left(\sum_{k \in z} f_{k} u^{k}\right):=\sqrt{\operatorname{Td}\left(T_{X}\right)} \sum_{k \in \mathbf{Z}} \operatorname{Ch}\left(f_{k}\right) u^{k}(z) .
$$

The factor $\sqrt{\operatorname{Td}\left(T_{X}\right)}$ is needed to match the cobordism-valued Poincaré pairing $\pi_{*}^{U}(a b)=\int_{X} \operatorname{Td}\left(T_{X}\right) \mathrm{Ch}(a) \mathrm{Ch}(b)$ with the cohomological one. The quantum Chern-Dold character provides a symplectic isomorphism of $\left(\mathcal{U}, \Omega^{U}\right)$ with $(\mathcal{H} \hat{\otimes} U, \Omega)$. This isomorphism identifies the Heisenberg Lie algebras and thus - due to the Stone-von Neumann Theorem - gives a projective identification of the corresponding Fock spaces. Understanding the quantum Chern-Dold character in this sense we obtain a family of "quantum states"

$$
\left\langle\mathcal{D}_{X}^{\mathrm{s}}\right\rangle:=\mathrm{qCh}\left\langle\mathcal{D}_{X}^{U}\right\rangle \text {. }
$$

These are 1-dimensional spaces depending formally on the parameter $\mathbf{s}=\left(s_{1}, s_{2}, \ldots\right)$ and spanned by asymptotic elements of the Fock space associated with $(\mathcal{H}, \Omega)$. We have $\left.\left\langle\mathcal{D}_{X}^{\mathbf{s}}\right\rangle\right|_{\mathbf{s}=\mathbf{0}}=\left\langle\mathcal{D}_{X}\right\rangle$. 
Introduce the virtual bundle $E=T_{X} \ominus \mathbf{C}$ over $X$, and identify $z \in H^{*}\left(\mathbf{C} P^{\infty}\right)$ with the equivariant first Chern class of the trivial line bundle $L$ over $X$ equipped with the standard fiberwise $S^{1}$-action.

Theorem (see [5]). Let $\hat{\Delta}$ be the quantization of the linear symplectic transformation $\Delta$ given by multiplication (in the algebra $\mathcal{H}$ ) by the asymptotic expansion of the infinite product

$$
\sqrt{\operatorname{Td}(E)} \prod_{m=1}^{\infty} \operatorname{Td}\left(E \otimes L^{-m}\right)
$$

Then

$$
\left\langle\mathcal{D}_{X}^{\mathbf{s}}\right\rangle=\hat{\Delta}(\mathbf{s})\langle\mathcal{D}\rangle
$$

We need to add to this formulation the following comments. The asymptotic expansion in question is obtained using the famous formula relating integration with its finite-difference version via the Bernoulli numbers:

$$
\begin{aligned}
\frac{s(x)}{2}+\sum_{m=1}^{\infty} s(x-m z) & =\frac{1+e^{-z d / d x}}{1-e^{-z d / d x}} \frac{s(x)}{2} \\
& \sim \sum_{m=0}^{\infty} \frac{B_{2 m}}{(2 m) !} z^{2 m-1} \frac{d^{2 m-1} s(x)}{d x^{2 m-1}} .
\end{aligned}
$$

Taking $s(x)=\sum_{k \geq 0} s_{k} x^{k} / k$ ! and leting $x$ run over Chern roots of $E$ we find that

$$
\ln \Delta=\sum_{m=0}^{\infty} \sum_{l=0}^{D} s_{2 m-1+l} \frac{B_{2 m}}{(2 m) !} \operatorname{ch}_{l}(E) z^{2 m-1},
$$

where $D=\operatorname{dim}_{\mathbf{C}}(X)$. The operators $A$ on $\mathcal{H}$ defined as multiplication by $\operatorname{ch}_{l}(E) z^{2 m-1}$ are infinitesimal symplectic transformations - they arer anti-symmetric with respect to $\Omega$ - and so define quadratic Hamiltonians $\Omega(A f, f) / 2$ on $\mathcal{H}$. We use the quantization rule of quadratic Hamiltonians written in a Darboux coordinate system $\left\{p_{\alpha}, q_{\alpha}\right\}$ :

$$
\left(q_{\alpha} q_{\beta}\right)^{\wedge}:=\frac{q_{\alpha} q_{\beta}}{\hbar},\left(q_{\alpha} p_{\beta}\right)^{\wedge}:=q_{\alpha} \frac{\partial}{\partial q_{\beta}},\left(p_{\alpha} p_{\beta}\right)^{\wedge}:=\hbar \frac{\partial^{2}}{\partial p_{\alpha} \partial p_{\beta}} .
$$

The rule defines the quantization $\hat{A}$ and the action on the quantum state $\left\langle\mathcal{D}_{X}\right\rangle$ of $\hat{\Delta}:=\exp (\ln \Delta)^{\wedge}$.

A more precise version of this Quantum-Hirzebruch-Riemann-Roch theorem provides also the proportionality coefficient between $\hat{\Delta} \mathcal{D}_{X}$ and 
$\mathcal{D}_{X}^{\mathrm{s}}$. Namely, let us write $\Delta=\Delta_{1} \Delta_{2}$ where $\ln \Delta_{2}$ consists of the $z^{-1}$ terms in (11) and $\ln \Delta_{1}$ contains the rest. Let us agree that $\hat{\Delta} \mathcal{D}_{X}$ means $\hat{\Delta}_{1} \hat{\Delta}_{2} \mathcal{D}_{X}$ (this is important since the two operators commute only up to a scalar factor). Then (see [5])

$$
\mathcal{D}_{X}^{\mathbf{s}}=(\operatorname{sdet} \sqrt{\operatorname{Td}(E)})^{1 / 24} e^{\frac{1}{24} \sum_{l>0} s_{l-1} \int_{X} \operatorname{ch}_{l}(E) c_{D-1}\left(T_{X}\right)} \hat{\Delta}(\mathbf{s}) \mathcal{D}_{X},
$$

where $c_{D-1}$ is the $(D-1)$-st Chern class, and sdet stands for Beresinian.

Taking the quasi-classical limit $\hbar \rightarrow 0$, we obtain the genus-zero version of the theorem. As in section 3, the graph of the differential $d \mathcal{F}_{0, X}^{U}$ of the cobordism-valued genus-zero potential defines a (formal germ of a) Lagrangian submanifold $\mathcal{L}_{X}^{U}$ of $\mathcal{U}$.

Corollary. The image $\mathrm{q} C h\left(\mathcal{L}_{X}^{U}\right)$ of the Lagrangian submanifold $\mathcal{L}_{X}^{U} \subset \mathcal{U}$ is obtained from the Lagrangian cone $\mathcal{L}_{X} \subset \mathcal{H}$ representing $d \mathcal{F}_{X}^{0}$ by the family of linear symplectic transformations $\Delta(\mathbf{s})$ :

$$
\mathrm{q} \operatorname{Ch}\left(\mathcal{L}_{X}^{U}\right)=\Delta \mathcal{L}_{X} .
$$

In particular, $\mathcal{L}_{X}^{U}$ is a Lagrangian cone with the property that its tangent spaces $T$ are tangent to $\mathcal{L}_{X}^{U}$ exactly along $u T$.

When $X=$ pt we have $\ln \Delta=-\sum_{m>0} s_{2 m-1} B_{2 m} z^{2 m-1} /(2 m)$ ! In the case $g=0$, since $\mathcal{L}_{\mathrm{pt}}$ is invariant under multiplication by $z$ we see that $\mathrm{qCh}\left(\mathcal{L}_{\mathrm{pt}}^{U}\right)=\mathcal{L}_{\mathrm{pt}}$. This is our Theorem from Section 3 .

\section{Outline of the proof of the QHRR theorem.}

The proof proceeds by showing that the derivatives in $s_{k}, k=$ $1,2, \ldots$, of the L.H.S. and the R.H.S. of (12) are equal. Differentiating the L.H.S. in $s_{k}$ brings down a factor of $\operatorname{ch}_{k}\left(T_{g, n}^{X, d}\right)$ inside all the correlators (10). Since the R.H.S. contains the generating function $\mathcal{D}_{X}$ for intersection numbers involving the classes $\psi_{i}$, our main problem now is to express the Chern character $\operatorname{ch}\left(T_{g, n}^{X, d}\right)$ in terms of $\psi$-classes.

One can view the moduli space $\overline{\mathcal{M}}_{g, n}(X, d)$ as fibered over the moduli stack $\overline{\mathfrak{M}}_{g, n}$ of marked nodal curves $\left(\Sigma ; \sigma_{1}, \ldots, \sigma_{n}\right)$. Thus the virtual tangent bundle $T_{g, n}^{X, d}$ falls into three parts:

$$
T_{g, n}^{X, d}=T^{\prime}+T^{\prime \prime}+T^{\prime \prime \prime}
$$

where $T^{\prime}$ is the virtual tangent bundle to the fibers, $T^{\prime \prime}$ is the (pullback of the) tangent sheaf to the moduli stack $\overline{\mathfrak{M}}_{g, n}$ logarithmic with respect to the divisor of nodal curves, and $T^{\prime \prime \prime}$ is a sheaf supported on the divisor. The subbundle $T^{\prime}$ - which is the index bundle of the Cauchy-Riemann operator describing infinitesimal variations of pseudo-holomorphic maps to $X$ from a fixed complex curve $\Sigma$ with a fixed configuration of marked points — can alternatively be described 
in terms of the twisting bundles considered in [6]. Namely, the diagram formed by the forgetting map $\mathrm{ft}_{n+1}: \overline{\mathcal{M}}_{g, n+1}(X, d) \rightarrow \overline{\mathcal{M}}_{g, n}(X, d)$ and the evaluation map $\operatorname{ev}_{n+1}: \overline{\mathcal{M}}_{g, n+1}(X, d) \rightarrow X$ can be considered as the universal family of genus- $g, n$-pointed stable maps to $X$ of degree $d$. Let $E$ be a complex bundle (or virtual bundle) over $X$. The K-theoretic pull-back/push-forward $E_{g, n}^{X, d}:=\left(\mathrm{ft}_{n+1}\right)_{*} \mathrm{ev}_{n+1}^{*}(E)$ is an element, called a twisting bundle, of the Grothendieck group of orbibundles $K^{*}\left(\overline{\mathcal{M}}_{g, n}(X, d)\right)$. The virtual bundle $T^{\prime}$ coincides with the twisting bundle $(T X)_{g, n}^{X, d}$.

Intersection numbers in $\overline{\mathcal{M}}_{g, n}(X, d)$ against characteristic classes of twisting bundles $E_{g, n}^{X, d}$ are called Gromov-Witten invariants of $X$ twisted by $E$. The "Quantum Riemann-Roch" theorem of [6] expresses such twisted Gromov-Witten invariants in terms of untwisted ones. The key to this is an application of the Grothendieck-Riemann-Roch theorem to the universal family $\mathrm{ft}_{n+1}: \overline{\mathcal{M}}_{g, n+1}(X, d) \rightarrow \overline{\mathcal{M}}_{g, n}(X, d)$, analogous to Mumford's famous computation [14] of the Hodge classes in $\overline{\mathcal{M}}_{g, 0}$ (and to its generalization to $\overline{\mathcal{M}}_{g, n}(X, d)$ by Faber and Pandharipande [7]). Applying the same idea here allows us to express the $\operatorname{classes} \mathrm{ch}_{k}\left(T^{\prime}\right)$ in terms of $\psi$-classes on the universal family $\overline{\mathcal{M}}_{g, n+1}(X, d)$.

Next, fibers of the logarithmic tangent bundle $T^{\prime \prime}$ can be viewed as dual to spaces of quadratic differentials on $\Sigma$ twisted appropriately at the marked points and nodes. More precisely, $T^{\prime \prime}=-\left(\mathrm{ft}_{n+1}\right)_{*}\left(L_{n+1}^{-1}\right)$, where $L_{n+1}$ is the universal cotangent line at the $(n+1)$ st marked point and $\left(\mathrm{ft}_{n+1}\right)_{*}$ is the K-theoretic push-forward. Thus by applying the Grothendieck-Riemann-Roch formula again we can $\operatorname{express} \operatorname{ch}_{k}\left(T^{\prime \prime}\right)$ in terms of $\psi$-classes on the universal family $\overline{\mathcal{M}}_{g, n+1}(X, d)$.

Finally, the sheaf $T^{\prime \prime \prime}$ can be expressed as the K-theoretic pushforward $\left(\mathrm{ft}_{n+1}\right)_{*} \mathcal{O}_{Z}$ of the structure sheaf of the locus $Z \subset \overline{\mathcal{M}}_{g, n+1}(X, d)$ of nodes of the curves $\Sigma$. This has (virtual) complex codimension 2 in the universal family $\overline{\mathcal{M}}_{g, n+1}(X, d)$. It is parametrized by certain pairs of stable maps with genera $g_{1}$ and $g_{2}$ where $g_{1}+g_{2}=g$, each of which carry an extra marked point, and by stable maps of genus $g-1$ which carry two extra marked points; the extra marked points are glued to form the node. Intersection numbers involving the $\operatorname{classes} \operatorname{ch}_{k}\left(T^{\prime \prime \prime}\right)$ can therefore be expressed in terms of intersections against $\psi$-classes in (products of) "simpler" moduli spaces of stable maps.

Together, the previous three paragraphs give recursive formulas which reduce intersection numbers involving the classes $\operatorname{ch}_{k}\left(T_{g, n}^{X, d}\right)$ to those involving only $\psi$-classes. Processing the $s_{k}$-derivative of the L.H.S. of (12) in this way one finds, after some 20 pages of miraculous cancellations and coincidences, that it is equal to the $s_{k}$-derivative of the R.H.S. We 
do not have any conceptual explanation for these cancellations, which often look quite surprising. For example, it turns out to be vital that the orbifold Euler characteristic $\chi\left(\overline{\mathcal{M}}_{1,1}\right)$ is equal to $5 / 12$ (or, equivalently, that $\left.c_{1}\left(T_{1,1}^{\mathrm{pt}, 0}\right)=10 \psi_{1}\right)$. Were this not the case, a delicate cancellation involving the cocycle coming from the projective representation of the Heisenberg Lie algebra would not have occurred, and the multiplicativity of (12) with respect to the group $\operatorname{Td}(\cdot)=\exp \sum s_{k} \operatorname{ch}_{k}(\cdot)$ of characteristic classes would have been destroyed.

The three summands $T^{\prime}, T^{\prime \prime}$, and $T^{\prime \prime \prime}$ play differing roles in the ultimate formula, as we now explain. Comparing the QHRR theorem with the Quantum Riemann-Roch theorem from [6], one sees that the potentials $\mathcal{D}_{X}^{\text {s }}$ coincide with the total potentials of $X$ for cohomological Gromov-Witten theory twisted by the characteristic class $\operatorname{Td}(\cdot)=\exp \sum s_{k} \operatorname{ch}_{k}(\cdot)$ and the bundle $E=T X-\mathbf{C}$. But the total potential $\mathcal{D}_{X}^{U}$ for cobordism-valued Gromov-Witten theory of $X$ differs from $\mathcal{D}_{X}^{\mathbf{s}}$ precisely because of the additional s-dependence coming from the quantum Chern-Dold character, i.e. through the s-dependence of the dilaton shift $u(-z)$ and of the polarization $\mathcal{H}_{+} \oplus \mathrm{qCh}\left(\mathcal{U}_{-}\right)$. These

effects are compensated for by $\left(\mathrm{ft}_{n+1}\right)_{*}\left(\mathbf{C}-L_{n+1}^{-1}\right)$ and $\left(\mathrm{ft}_{n+1}\right)_{*}\left(\mathrm{O}_{Z}\right.$ respectively. Thus, roughly speaking, the quantized symplectic transformation $\hat{\Delta}$ accounts for variations $T^{\prime}$ of maps $\Sigma \rightarrow X$, while the symplectic formalism of Section 3 based on formal groups accounts for variations $T^{\prime \prime}+T^{\prime \prime \prime}$ of complex structures on $\Sigma$. This suggests that there is an intrinsic relationship between formal group laws and the moduli space of Riemann surfaces. However, the precise nature of this relationship remains mysterious.

\section{REFERENCES}

[1] S. Barannikov. Quantum periods - I. Semi-infinite variations of Hodge structures. Preprint, alg-geom/0006193.

[2] K. Behrend, B. Fantechi. The intrinsic normal cone. Invent. Math. 128 (1997), $45-88$.

[3] K. Behrend, Yu. Manin. Stacks of stable maps and Gromov-Witten invariants. Duke Math. J. 85 (1996), 1 - 60.

[4] V. M. Bukhshtaber. The Chern-Dold character in cobordisms. Mat. Sb. (N.S.) 83 (1970), 575-595.

[5] T. Coates. Riemann - Roch theorems

in Gromov - Witten theory. PhD thesis, UC Berkeley, 2003. http://abel.math.harvard.edu/ tomc/

[6] T. Coates and A. Givental. Quantum Riemann - Roch, Lefschetz and Serre. arXiv: math.AG/0110142

[7] C. Faber, R. Pandharipande. Hodge integrals and Gromov - Witten theory. Invent. Math. 139 (2000), 173-199. 
[8] K. Fukaya, K. Ono. Arnold conjecture and Gromov-Witten invariants. Topology 38 (1999), no. 5, 933-1048.

[9] A. Givental. Symplectic geometry of Frobenius structures. To appear in Proceedings of the workshop on Frobenius structures (MPI-Bonn, July 2002), C. Hertling, Yu. Manin, M. Marcolli (eds.) arXiv: math.AG/0305409.

[10] A. Givental. Gromov - Witten invariants and quantization of quadratic hamiltonians. Moscow Mathematical Journal, v.1(2001), no. 4, 551-568.

[11] M. Kontsevich Intersection theory on the moduli space of curves and the matrix Airy function. Commun. Math. Phys. 147 (1992), 1 - 23.

[12] M. Kontsevich. Enumeration of rational curves via toric actions. In: The Moduli Space of Curves (R, Dijkgraaf, C. Faber, G. van der Geer, eds.) Progr. in Math. 129, Birkhäuser, Boston, 1995, 335-368. M. Kontsevich.

[13] J. Li, G. Tian. Virtual moduli cycles and Gromov-Witten invariants of algebraic varieties. J. Amer. Math. Soc. 11 (1998), no. 1, 119-174.

[14] D. Mumford. Towards enumerative geometry on the moduli space of curves. In: Arithmetics and Geometry (M. Artin, J. Tate eds.), v.2, Birkhäuser, 1983, $271-328$.

[15] Y. Ruan. Virtual neighborhoods of pseudo-holomorphic curves. Proceedings of 6th Gkova Geometry-Topology Conference. Turkish J. Math. 23 (1999), no. $1,161-231$.

[16] B. Siebert. Algebraic and symplectic Gromov-Witten invariants coincide. Ann. Inst. Fourier (Grenoble), 49 (1999), No. 6, 1743-1795.

[17] E. Witten. Two-dimensional gravity and intersection theory on moduli space. Surveys in Diff. Geom. 1 (1991), 243-310.

Dept. of Math., Harvard University, Boston, MA 02138, USA

Dept. of Math., University of California Berkeley, CA 94720, USA 